\title{
Generalised Equilibrium Reduced-Density Operator
}

\author{
Z. JACYNA-ONYSZKIEWICZ* \\ Quantum Physics Division, Faculty of Physics, Adam Mickiewicz University, Umultowska 85, 61-614 Poznań, Poland
}

(Received September 8, 2017; in final form February 19, 2018)

Equilibrium reduced-density operators for subsystems of the universe are derived from the generalised Schrödinger variational principle, additionally assuming that the values of von Neumann entropy for the subsystems are fixed and higher than zero. The obtained reduced-density operator may be useful for description of the properties of an arbitrary system (macroscopic, mesoscopic, nanoscopic, or microscopic) in the equilibrium mixed state.

DOI: 10.12693/APhysPolA.133.1231

PACS/topics: 03.65.Ca, 05.30.-d

\section{Introduction}

Usually, when investigating theoretically macroscopic bulk systems, we do not have to take into account their interaction with environment, regarding the short-range character of intermolecular interactions. Any effective interaction among the molecules of the system and its environment could only occur through the atoms on or near the system's surface. The number of interacting atoms is usually an insignificant part of all atoms of the system. However, considering ultrathin films and similar systems, the number of atoms interacting with environment is frequently of the same order of magnitude as the total number of atoms in the system. Moreover, thin films are deposited on bulk substrates whose structure affects that of the films, so the interaction between the substrate and the film has to be taken into account. A common procedure to realise it is to introduce phenomenological surface parameters. The values of these parameters significantly influence physical properties of mesoscopic systems or thin films, which for example is evidenced in their spectra of collective excitations.

For such system the postulates of the quantum statistical thermodynamics (QST) are not exactly fulfilled as this theory assumes that the effect of environment can only be a mixing of quantum states in the system studied and it has no effect on the spectrum of eigenstates of its Hamiltonian.

In this paper we propose a general method which takes into account the interaction between the nanoscopic or mesoscopic system or thin film and the substrate on a microscopic level. In this method, the interactions are included already in the construction of the reduced density operator. The method proposed is more general than the QST and is an extension of the ideas presented earlier [1-3].

\section{Statement of the problem}

Let us consider a closed quantum system composed of $N$-particles in a stationary state of a fixed energy $E$.

*e-mail: zbigonys@amu.edu.pl
Assume that the energy of the system has been determined by macroscopic methods with an accuracy not better than $\pm \delta E$. For the sake of simplicity let us assume that the system is composed of $N$ identical weakly interacting particles (weakly enough so that the interaction does not significantly change the spectrum of states of individual particles). Each of the particles can be in one of $\Gamma$ states, while the total energy of the system is within the range $E \pm \delta E$. Thus, the number of the states of the system can be estimated as $\Gamma^{N}$, and the ratio of the mean distance between the states on the energy axis $\delta \bar{E}$ to the accuracy of energy measurement is

$$
\frac{\delta \bar{E}}{\delta E} \approx \frac{2}{\Gamma^{N}} .
$$

As follows from this estimation, the mean distance on the energy axis between the neighbouring stationary states of the system for which $N \gg 1$ is very small relative to $\delta E$, so that even a minimum external disturbance can shift the system from one state to another. Such disturbances are always present, for instance as a result of unscreened gravitation interactions. That is why the macroscopic massive systems cannot be treated as isolated from environment and thus describable in terms of quantum theory formalism but by QST. The only macroscopic massive system with $N \gg 1$, which can be treated as a closed and fully isolated one is the whole universe. All other macroscopic bulk systems must be considered as non-isolated so interacting with environment.

The idea of description of the universe as a closed system in terms of quantum theory has created a quantum cosmology. In quantum cosmology the universe is understood as the largest physical system with no external physical reality, which can be described in the quantum theory. In quantum cosmology attempts are made to describe the universe in the categories of the state vectors. The quantum cosmology deals with close universe model (universe with a finite volume), because, in the quantum theory, only such a universe can be described consistently. The total energy of such a universe is exactly zero and the state vector of the universe cannot depend on time [4]. The positive energy of the universe matter and dark energy is exactly compensated by the negative energy connected with the gravitation. 
Therefore, the equation for the state vector of the close universe $|\psi\rangle$ is

$$
H|\psi\rangle=0 \text {, }
$$

where $H$ is the Hamiltonian of the universe.

In general, Eq. (2) has degenerate eigenvalues and the stationary state $\psi$ is not determined exactly by the value of energy $E$, but by a set of eigenvalues of all operators making a complete set of commutating operators with $H$ (CSCO).

The eigenequations for CSCO can be written as:

$$
\begin{aligned}
& Q_{1}\left|q_{1} q_{2} \ldots\right\rangle=q_{1}\left|q_{1} q_{2} \ldots\right\rangle, \\
& Q_{2}\left|q_{1} q_{2} \ldots\right\rangle=q_{2}\left|q_{1} q_{2} \ldots\right\rangle, \\
& \ldots \ldots \ldots \ldots \ldots \ldots \ldots \ldots, \ldots \ldots \ldots
\end{aligned}
$$

where $\mathrm{CSCO}=\left\{Q_{1}=H, Q_{2}, \ldots\right\}$.

Introducing the matrix notation the set of Eqs. (3) can be written in the compact form

$$
\underline{Q}|\underline{q}\rangle=\underline{q}|\underline{q}\rangle
$$

where $\underline{Q}$ represents $\mathrm{CSCO}$ in the form of a single-matrix column

$$
\underline{Q}:=\left\|\begin{array}{c}
Q_{1}=H \\
Q_{2} \\
\vdots
\end{array}\right\|=\left\{Q_{i}\right\},
$$

and $i=1,2, \ldots$ This matrix is known as the complete matrix of operators. The matrix $\underline{q}$ represents the set of eigenvalues of CSCO

$$
\underline{q}:=\left\|\begin{array}{c}
q_{1}=0 \\
q_{2} \\
\vdots
\end{array}\right\|=\left\{q_{i}\right\},
$$

and is known as the complete matrix of eigenvalues. Note that Eq. (4) has formally non-degenerate complete matrices of eigenvalues. After orthogonalisation of the state vectors $|\underline{q}\rangle$, they meet the conditions

$$
\begin{aligned}
& \left\langle\underline{q} \mid \underline{q}^{\prime}\right\rangle=\delta_{\underline{q}, \underline{q}^{\prime}}, \\
& \sum_{\underline{q}}|\underline{q}\rangle\langle\underline{q}|=1 .
\end{aligned}
$$

Neither any specific form of the complete matrix of operators $\underline{Q}$ nor a specific form of the vector of state $|q\rangle$ are relevant for us. We assume that the universe is in the stationary state characterised by the unknown state vector $|\underline{q}\rangle$. Equation (4) is a very simple but useful generalisation of the stationary Schrödinger Eq. (2).

It can be easily shown that Eq. (4) is equivalent to the generalised Schrödinger variational principle

$$
\delta \operatorname{Tr}(\underline{Q} d)=\underline{0}
$$

with the normalization condition for the density operator $d$ :

$$
\operatorname{Tr}(d)=1,
$$

where

$$
d=|\underline{q}\rangle\langle\underline{q}|,
$$

and the first variation of the density operator takes the form

$$
\delta d:=|\delta \underline{q}\rangle\langle\underline{q}|+| \underline{q}\rangle\langle\delta \underline{q}|,
$$

and is expressed in terms of the variation $|\delta q\rangle$ of the state vector. Using the Lagrange method for finding the conditional extreme of the functionals, Eqs. (9) and (10) can be rewritten in the form

$$
\delta \operatorname{Tr}((\underline{Q}-\underline{q}) d)=\underline{0},
$$

where $q$ is the complete matrix of indeterminate Lagrange multipliers.

The assumed stationary character of the universe implies the constant in time expectation values of any observables $a$ :

$$
\langle a\rangle=\langle\underline{q}|a| \underline{q}\rangle=\operatorname{Tr}[a d] .
$$

For this reason we shall restrict our considerations to the many-particle systems being in such an environment and in such a state that the time changes in these systems are irrelevant, so we shall study the systems only in the equilibrium states.

\section{Equilibrium reduced-density operator}

Let us divide the universe into $k$ arbitrary systems $j=1,2, \ldots, k$ in such a way that each of them would satisfy the equilibrium condition in a good approximation. We have experienced that the presence of a many particle system in a state which can be in a good approximation treated as equilibrium is nothing unusual. It is always possible to divide the universe into at least two parts $(k=2)$ : a given system $j$ being in the equilibrium state and the other part of the universe $R$. If a given system is in the equilibrium state, its environment cannot significantly change in time. Therefore, we can reasonably assume that also the other part of the universe is in the equilibrium state.

Analysis of each specific physical problem requires a proper, intuitively guessed, division of the universe into $k$ parts. Although this division is in a sense arbitrary, it does not change the generality of our considerations.

The complete matrix of the universe operators $Q$ can be in general written as

$$
\underline{Q}=\sum_{j=1}^{k} \underline{Q}_{j}+\sum_{j \neq j^{\prime}} \underline{Q}_{j j^{\prime}}+\underline{\tilde{Q}},
$$

where $\underline{Q}_{j}$ is the matrix of operators of the $j$-th system and $\underline{Q}_{j j^{\prime}}$ is the matrix of operators describing the interaction between the systems $j$ and $j^{\prime}$. Moreover

$$
\underline{Q}=\left\|Q_{1 j j^{\prime}}=H_{j j^{\prime}}\right\| \quad \underline{\tilde{Q}}=\| \begin{gathered}
0 \\
0 \\
0 \\
\vdots \\
0
\end{gathered}
$$

where $H_{j j^{\prime}}$ is the Hamiltonian describing the interactions 
among these systems and $\tilde{Q}$ is the matrix of operators of non-additive observables of the universe.

According to Eq. (14), the expectation value of an arbitrary observable $a_{j}$ of the system $j$ is

$$
\left\langle a_{j}\right\rangle=\operatorname{Tr}\left(a_{j} d\right) \text {. }
$$

The calculation of the trace in Eq. (17) can be performed at two stages. At first we realise that the following equality holds:

$$
\left\langle a_{j}\right\rangle=\operatorname{Tr}_{j}\left(a_{j} d_{j}\right),
$$

where

$$
d_{j}=\operatorname{Tr}_{R}(d)
$$

is the reduced-density operator [5] of the system $j$ and $\operatorname{Tr}_{R}[\ldots]$ is the partial trace over the states of the other part of the universe $R$.

Although the universe as a whole is in the pure state $|q\rangle$, its arbitrary macroscopic many-particle parts inevitably are in mixed states. This is a purely quantum effect following from the holistic properties of the quantum theory formally related to the fact that the universe (according to the quantum cosmology postulates) has one vector of state common for all systems and in the case of interactions among them we are not able to specify the vector of state for an individual system $j$ (it may not be the case for the interactions of the effective field type). Such a situation does not occur in the classical description because we can know classical trajectories of each particular molecule irrespective of their interactions. The holistic features of the quantum theory imply the use of the formalism of the reduced-density operators in description of many particle systems.

The dimensionless von Neumann entropy for a system $j$, defined by the equation:

$$
s_{j}:=-\operatorname{Tr}_{j}\left(d_{j} \ln d_{j}\right)
$$

takes the highest value for a homogeneous distribution, that is the one in which the presence of the system $j$ in any eigenstate of the reduced density operator $d_{j}$ is equally probable. Such a state can be described as having the greatest degree of state mixing. In the pure state, without state mixing, the entropy takes the smallest value equal to zero. Therefore, the dimensionless entropy is a good, additive measure of the degree of state mixing.

Our main assumption concerning the equilibrium state of any system $j$ can be expressed as

$$
\underset{j}{\forall s_{j}}=\text { constant }>0 \text {. }
$$

This postulate takes into account the fact that none bulk macroscopic many-particle system other than the universe can be treated as closed and so all other macroscopic systems have to be in a mixed state.

On the other hand, this postulate may not hold for the equilibrium systems containing a very small number of particles, for example in ion trap, because such systems can be in a pure quantum state whose entropy is $s_{j}=0$. Therefore, systems of a very small number of particles can have their attributed state vectors and can be described in terms of quantum mechanics so without any statistical hypotheses that are beyond the quantum theory.

We assume that when there is an interaction between any $k$ systems, the operator of density of the universe $d$ can always be expressed as

$$
d:=K \underset{j=1}{\otimes} d_{j},
$$

where $K$ is the correlation superoperator [6] acting in the space of the reduced density operators (in general a superoperator is a symbol of the mathematical operation by which an operator is formed from another operator). Moreover we assume that the superoperator $K$ is not a function of density operators, so

$$
K \neq K\left(d_{1}, d_{2}, \ldots, d_{k}\right) .
$$

For our considerations important is the existence of such a superoperator and not its actual form. The existence of such a superoperator can be proven on very simple examples. Of course it cannot be shown to exist for a certain division of the universe into $k$ parts. Therefore, we have to assume the existence of the correlation superoperator $K$ defined by (22) and satisfying the condition (23).

Our basic assumptions on the state vector of the universe $|q\rangle$ can be expressed as the generalised Schrödinger variation principle (13):

$$
\delta \operatorname{Tr}((\underline{Q}-\underline{q}) d)=\underline{0}
$$

with an additional condition following from formula (21), i.e.

$$
s_{j}=\text { constant }>0
$$

for $j=1,2, \ldots, k$ where $q$ is the complete matrix of indeterminate Lagrange multipliers. According to this requirement each of the $k$ systems into which the universe was divided is in an equilibrium mixed state. The variation principle (24) together with (25) express our main assumptions.

Applying the Lagrange method for the calculation of the conditional extremes of the functionals, we can write these two expressions as

$$
\delta\left(\operatorname{Tr}((\underline{Q}-\underline{q}) d)+\sum_{j=1}^{k} \underline{A}_{j} s_{j}\right)=\underline{0},
$$

where $\underline{A}_{j}$ are the single column matrices of the indeterminate Lagrange multipliers.

Substituting Eqs. (15), (22) and (20) into (26) we arrive at

$$
\begin{gathered}
\delta\left(\sum_{j=1}^{k} \operatorname{Tr}\left(\underline{Q}_{j} d\right)+\operatorname{Tr}\left(\left(\sum_{j \neq j^{\prime}} \underline{Q}_{j j^{\prime}}+\underline{\tilde{Q}}\right) K \underset{j^{\prime \prime}=1}{\otimes} d_{j^{\prime \prime}}\right)\right. \\
\left.-\underline{q} \operatorname{Tr}\left(K \underset{j^{\prime \prime}=1}{\otimes} d_{j^{\prime \prime}}\right)-\sum_{j=1}^{k} \underline{A}_{j} \operatorname{Tr}_{j}\left(d_{j} \ln d_{j}\right)\right)=\underline{0} .
\end{gathered}
$$

Taking into account the definition of the reduceddensity operator (19) and Eq. (21) we get 


$$
\begin{aligned}
& \sum_{j=1}^{k}\left(\operatorname{Tr}_{j}\left(\underline{Q}_{j} \delta d_{j}\right)+\operatorname{Tr}_{j}\left(\underline{Q}_{j}^{\prime} \delta d_{j}\right)\right. \\
& \left.\quad-\underline{A}_{j} \operatorname{Tr}_{j}\left(\delta d_{j} \ln d_{j}+\delta d_{j}\right)-\underline{q}^{\operatorname{Tr}}\left(\delta d_{j}\right)\right)=\underline{0},
\end{aligned}
$$

where

$$
\underline{Q}_{j}^{\prime}=\operatorname{Tr}_{R}\left(\left(\sum_{j^{\prime}} \underline{Q}_{j j^{\prime}}+\underline{\tilde{Q}}\right) K \underset{j^{\prime \prime} \neq j}{\otimes} d_{j^{\prime \prime}}\right) .
$$

Moreover, as follows from the normalization conditions (10), (19) and from

$$
\begin{aligned}
& \operatorname{Tr}\left(K \underset{j "=1}{\otimes} d_{j \prime}\right)=1 \\
& \delta d_{j}=\delta \operatorname{Tr}_{R}\left(K \underset{j^{\prime \prime}=1}{\otimes} d_{j^{\prime \prime}}\right)=\delta\left(\operatorname{Tr}_{R}\left(K \underset{j^{\prime \prime} \neq j}{\otimes} d_{j^{\prime \prime}}\right) d_{j}\right)= \\
& \delta\left(1_{j} d_{j}\right)=\delta d_{j},
\end{aligned}
$$

where $\operatorname{Tr}_{R}(\ldots)$ is the partial trace over the states of the universe without the system $j$. Then we have

$\sum_{j=1}^{k} \operatorname{Tr}_{j}\left(\left(\underline{Q}_{j}+\underline{Q}_{j}^{\prime}-\underline{A}_{j} \ln d_{j}-\underline{A}_{j}-\underline{q}\right) \delta d_{j}\right)=\underline{0}$.

Because of the arbitrariness of variations $\delta d_{j}$ of the reduced density operator $d_{j}$, we get

$$
\ln d_{j}=-\underline{A}_{j}^{-1} \underline{q}-1+\underline{A}_{j}^{-1}\left(\underline{Q}_{j}+\underline{Q}_{j}^{\prime}\right),
$$

where $\underline{A}_{j}^{-1}$ is the inverse matrix of $\underline{A}_{j}$, i.e.

$$
\underline{A}_{j}^{-1} \underline{A}_{j}=1 \text {. }
$$

Introducing new notation

$$
\begin{aligned}
& L_{j}=1+\underline{A}_{j}^{-1} \underline{q}, \\
& \underline{P}_{j}=-\underline{A}^{-1},
\end{aligned}
$$

we finally arrive at the formula for the reduced-density operator of the $j$-th system in the equilibrium state

$$
d_{j}=\exp \left(-L_{j}-\underline{P}_{j}\left(\underline{Q}_{j}+\underline{Q}_{j}^{\prime}\right)\right) .
$$

From the normalization condition we can calculate the indeterminate Lagrange multipliers

$$
L_{j}=\ln \operatorname{Tr}_{j}\left(\exp \left(-\underline{P}_{j}\left(\underline{Q}_{j}+\underline{Q}_{j}^{\prime}\right)\right)\right) .
$$

and putting (37) into (20) we get the following expression for the dimensionless entropy of the $j$-th system:

$$
s_{j}=L_{j}+\underline{P}_{j}\left\langle\underline{Q}_{j}+\underline{Q}_{j}^{\prime}\right\rangle,
$$

where

$$
\begin{aligned}
& \left\langle\underline{Q}_{j}+\underline{Q}_{j}^{\prime}\right\rangle= \\
& \quad \operatorname{Tr}_{j}\left(\left(\underline{Q}_{j}+\underline{Q}_{j}^{\prime}\right) \exp \left(-L_{j}-\underline{P}_{j}\left(\underline{Q}_{j}+\underline{Q}_{j}^{\prime}\right)\right)\right) .
\end{aligned}
$$

Having calculated $L_{j}$ from (39) and putting it into (37) we arrive at another form of the reduced density operator of the $j$-th system

$$
d_{j}=\exp \left(-s_{j}-\underline{P}_{j} \Delta \underline{Q}_{j}-\underline{P}_{j} \Delta \underline{Q}_{j}^{\prime}\right),
$$

where

$$
\begin{aligned}
& \Delta \underline{Q}_{j}:=\underline{Q}_{j}-\left\langle\underline{Q}_{j}\right\rangle, \\
& \Delta \underline{Q}_{j}^{\prime}:=\underline{Q}_{j}^{\prime}-\left\langle\underline{Q}_{j}^{\prime}\right\rangle
\end{aligned}
$$

are the respective fluctuation operators.

Then, using Eq. (18), we arrive at the formula for the expectation value of any observable of the $j$-th system

$$
\begin{gathered}
\left\langle a_{j}\right\rangle=\operatorname{Tr}_{j}\left(a_{j} \exp \left(-L_{j}-\underline{P}_{j} \underline{Q}_{j}-\underline{P}_{j} \underline{Q}_{j}^{\prime}\right)\right)= \\
\operatorname{Tr}_{j}\left(a_{j} \exp \left(-s_{j}-\underline{P}_{j} \Delta \underline{Q}_{j}-\underline{P}_{j} \Delta \underline{Q}_{j}^{\prime}\right)\right) .
\end{gathered}
$$

The mean fluctuation of the non-additive observables is equal to zero [3]. This means that the non-additive values cannot be important. Hence, finally the general expression for the reduced-density operator for the $j$-th system is

$$
\begin{gathered}
d_{j}=\exp \left(-L_{j}-\underline{P}_{j}\left(\underline{Q}_{j}+\underline{Q}_{j}^{\prime}\right)\right)= \\
\exp \left(-s_{j}-\underline{P}_{j}\left(\Delta \underline{Q}_{j}+\Delta \underline{Q}_{j}^{\prime}\right)\right),
\end{gathered}
$$

where now

$$
\underline{Q}_{j}^{\prime}=\sum_{j^{\prime} \neq j} \operatorname{Tr}_{R}\left(\underline{Q}_{j j^{\prime}} K \underset{j^{\prime \prime} \neq j}{\otimes} d_{j^{\prime \prime}}\right)
$$

and

$$
L_{j}=\operatorname{Tr}_{j}\left(\exp \left(-\underline{P}_{j}\left(\underline{Q}_{j}+\underline{Q}_{j}^{\prime}\right)\right)\right) .
$$

Putting (44) into (18) we get

$$
\left\langle a_{j}\right\rangle=\operatorname{Tr}_{j}\left(a_{j} \exp \left(-L_{j}-\underline{P}_{j}\left(\underline{Q}_{j}+\underline{Q}_{j}^{\prime}\right)\right)\right),
$$

where $\underline{Q}_{j}^{\prime}$ is described by Eq. (45) and $j=1,2, \ldots, k$. Using this expression we are able to describe an arbitrary physical system (macroscopic, mezoscopic, nanoscopic or microscopic) in the equilibrium mixed state.

\section{Particular cases of the reduced-density operators}

\subsection{Massive macroscopic system}

Let us assume that the $j$-th system is a massive macroscopic system (a system in which the number of surface particles is negligibly small when compared to the total number of particles) composed of $N_{j} \gg 1$ particles. In typical conditions, when the unscreened gravitational interaction is not dominant, the system interacts with its environment via short-range intermolecular forces. In this way the $j$-th system interacts with its environment only via the particles close to its surface. This means that the interaction with environment is a very small surface effect, which can be neglected. Thus, the reduced-density operator (44) becomes

$d_{j}=\exp \left(-L_{j}-\underline{P}_{j} \underline{Q}_{j}\right)=\exp \left(-s_{j}-\underline{P}_{j} \Delta \underline{Q}_{j}\right)$.

In the thermodynamical limit the terms containing the fluctuation operators are irrelevant. That is why for an 
infinitely large system we get a whole family of equivalent reduced-density operators. However, for the finite systems, depending on the specific physical conditions of the system $j$, particular components of the sum $\sum_{i} P_{i j} \Delta Q_{i j}$ (following from the matrix formalism) will bring a smaller or greater contribution to the operator $d_{j}$. Of course it will depend on whether the observable $Q_{i j}$ undergoes strong or weak fluctuations. Let us consider a few simple cases.

(i) Let us assume that the system $j$ is very well isolated from its environment and according to the macroscopic energy measurements its energy varies within a very narrow interval. Under this assumption we can assume that the matrix $\Delta \underline{Q}_{j}=\underline{0}$, which means that we neglect the fluctuations of all observables whose operators commute with the Hamiltonian $\mathrm{H}_{j}$. Thus, operator (48) takes the form

$$
d_{j}=\mathrm{e}^{-s_{j}} .
$$

In this case the operator $d_{j}$ is reduced to a real number. Let us write this operator in the basis of eigenvectors $\left|\underline{q}_{j}\right\rangle$ of the complete operator $\underline{Q}_{j}$ so in the basis of the vectors satisfying the usual conditions

$$
\begin{aligned}
& \underline{Q}_{j}\left|\underline{q}_{j}\right\rangle=\underline{q}_{j}\left|\underline{q}_{j}\right\rangle, \\
& \left\langle\underline{q}_{j} \mid \underline{q}_{j}^{\prime}\right\rangle=\delta_{\underline{q}_{j}, \underline{q}_{j}^{\prime}}, \\
& \sum_{\underline{q}_{j}}\left|\underline{q}_{j}\right\rangle\left\langle\underline{q}_{j}\right|=1 .
\end{aligned}
$$

Not all states $\left|\underline{q}_{j}\right\rangle$ can be realised because the energy of the system $j$ varies within a narrow interval $\delta E$. Therefore, the sum (52) can include only such states $\left|\underline{q}_{j}\right\rangle \in \delta E$, whose energy falls within this range. Thus we get

$$
d_{j}=\mathrm{e}^{-s_{j}} \sum_{\underline{q}_{j} \in \delta E}\left|\underline{q}_{j}\right\rangle\left\langle\underline{q}_{j}\right| .
$$

This operator has to satisfy the normalisation condition (28), so

$$
d_{j}=\frac{1}{w_{j}} \sum_{\underline{q}_{j} \in \delta E}\left|\underline{q}_{j}\right\rangle\left\langle\underline{q}_{j}\right|
$$

and

$$
s_{j}=\ln w_{j},
$$

where

$$
w_{j}=\sum_{\underline{q}_{j} \in \delta E}
$$

is the number of states allowed for the system $j$.

The reduced-density operator (54) is equivalent to the statistical operator for the microcanonical Gibbs distribution in QST. This operator has been obtained by a different path than it is done in the statistical thermodynamics. It has a simple form but in practice it is rarely applied because its use requires a thorough examination of the set of eigenstates of the system $j$, which is usually very difficult. (ii) More often used is the operator in which only the possibility of fluctuation of the system's $j$ energy is considered. It means that the system $j$ can exchange energy with its environment. In this case operator (48) takes the form

$$
d_{j}=\mathrm{e}^{-s_{j}-P_{1 j} \Delta Q_{1 j}},
$$

where

$$
\Delta Q_{1 j}=Q_{1 j}-\left\langle Q_{1 j}\right\rangle=H_{j}-\left\langle H_{j}\right\rangle
$$

and $H_{j}$ is the Hamiltonian of the system $j$.

This operator can be expressed as

$$
d_{j}=\mathrm{e}^{-L_{j}-P_{1 j} Q_{1 j}}=e^{\beta_{j}\left(F_{j}-H_{j}\right)},
$$

where

$$
\begin{aligned}
& L_{j}=-\beta_{j} F_{j}, \\
& P_{1 j}=\beta_{j}
\end{aligned}
$$

and from the normalization condition

$$
\begin{aligned}
& F_{j}=-\frac{1}{\beta_{j}} \ln Z_{j}, \\
& Z_{j}=\operatorname{Tr}_{j}\left(\mathrm{e}^{-\beta_{j} H_{j}}\right) .
\end{aligned}
$$

The form of operator (59) is the same as that of the statistical operator of the Gibbs canonical distribution in QST for the system $j$, and the parameter $Z_{j}$ is known as the canonical partition function. Referring to the commonly applied thermodynamics $[6,7]$ for a physical interpretation of $F_{j}$ and $\beta_{j}$, we find that $F_{j}$ is the free energy and $\beta_{j}=\left(k_{\mathrm{B}} T\right)^{-1}$, where $k_{\mathrm{B}}$ is the Boltzmann constant and $T$ is the absolute temperature.

(iii) Let us assume that the system $j$ can exchange not only energy but also $n$ types of particles with its environment. For each type of particles we can define an operator of the number of particles $N_{j m}$, where $m=2, \ldots, n+1$, and we moreover assume that

$$
N_{j m}\left|\underline{q}_{j}\right\rangle=n_{j m}\left|\underline{q}_{j}\right\rangle \text {, }
$$

where $n_{j m}$ is the number of particles of type $m$. Under the above assumptions the numbers of molecules are not constant and can fluctuate, thus operator (48) takes the form

$$
d_{j}=\exp \left(-s_{j}-P_{1 j} \Delta Q_{1 j}-\sum_{m=2}^{n+1} P_{m j} \Delta Q_{m j}\right),
$$

where

$$
\Delta Q_{m j}=Q_{m j}-\left\langle Q_{m j}\right\rangle=N_{j m}-\left\langle N_{j m}\right\rangle .
$$

This operator can be expressed as

$$
d_{j}=\exp \left(\beta_{j}\left(\Omega_{j}-H_{j}+\sum_{m=2}^{n+1} \mu_{j m} N_{j m}\right)\right),
$$

where

$$
\Omega_{j}=-\frac{1}{\beta_{j}} \ln \tilde{Z}_{j}
$$

and

$$
\tilde{Z}_{j}=\operatorname{Tr}_{j}\left(\exp \left(-\beta_{j}\left(H_{j}-\sum_{m=2}^{n+1} \mu_{j m} N_{j m}\right)\right)\right) .
$$


The form of operator (67) is the same as that of the statistical operator for the grand canonical Gibbs distribution in QST and $\tilde{Z}_{j}$ is the grand canonical partition function $[6,7]$.

The above three reduced-density operators and the equivalent statistical operators can be applied for description of a majority of the massive macroscopic systems. They make the basis of the QST. It should be noted that they are particular cases of the generalised reduceddensity operator (44).

\subsection{Microscopic exactly solvable spin systems}

We have shown that for macroscopic systems in the equilibrium state the reduced-density operator (44) leads to the results identical with those of QST. On the example of an exactly solvable simple microscopic system we will show that the reduced-density operator (44) leads to exact results.

Let us consider an isolated microscopic system composed of two spins $S_{1}$ and $S_{2}$, described by the anisotropic Heisenberg model $(s=1 / 2)$ whose Hamiltonian is

$$
H=\sum_{r} J_{r} S_{1}^{r} S_{2}^{r},
$$

where $r=x, y, z$ and $J_{x}=J_{y}=J, J_{z}=J^{\prime}$ are the exchange parameters. The eigenequations for the $z$ component of particular spins are of the form

$$
\begin{aligned}
& S_{1}^{z}|s\rangle_{1}=\frac{1}{2} \hbar s|s\rangle_{1}, \\
& S_{2}^{z}|s\rangle_{2}=\frac{1}{2} \hbar s|s\rangle_{2},
\end{aligned}
$$

where $s= \pm 1$.

Solving the stationary Schrödinger equation

$$
H|E\rangle=E|E\rangle,
$$

we get the following four solutions:

$$
\begin{aligned}
\left|E_{1}\right\rangle & =|1\rangle_{1} \otimes|1\rangle_{2}, \quad E_{1}=\frac{\hbar^{2}}{4} J^{\prime} \\
\left|E_{2}\right\rangle & =|-1\rangle_{1} \otimes|-1\rangle_{2}, \quad E_{2}=E_{1} \\
\left|E_{3}\right\rangle & =\frac{1}{\sqrt{2}}\left(|1\rangle_{1} \otimes|-1\rangle_{2}+|-1\rangle_{1} \otimes|1\rangle_{2}\right), \\
& E_{3}=\frac{\hbar^{2}}{4}\left(2 J-J^{\prime}\right), \\
\left|E_{4}\right\rangle & =\frac{1}{\sqrt{2}}\left(|1\rangle_{1} \otimes|-1\rangle_{2}-|-1\rangle_{1} \otimes|1\rangle_{2}\right), \\
& E_{4}=\frac{\hbar^{2}}{4}\left(-2 J-J^{\prime}\right) .
\end{aligned}
$$

Let us assume for example that the system considered is in the state $\left|E_{3}\right\rangle$. From Eq. (19) we can find exactly the reduced density operators $\tilde{d}_{1}$ and $\tilde{d}_{2}$ for the first and second spin, respectively. We arrive at

$$
\begin{aligned}
& \tilde{d}_{1}=\operatorname{Tr}_{2}(d)=\frac{1}{2}|-1\rangle_{1}{ }_{1}\left\langle-1\left|+\frac{1}{2}\right| 1\right\rangle_{1}{ }_{1}\langle 1|, \\
& \tilde{d}_{1}=\operatorname{Tr}_{1}(d)=\frac{1}{2}|-1\rangle_{2}{ }_{2}\left\langle-1\left|+\frac{1}{2}\right| 1\right\rangle_{2}{ }_{2}\langle 1|,
\end{aligned}
$$
where

$$
d=\left|E_{3}\right\rangle\left\langle E_{3}\right| \text {. }
$$

Let us now calculate the reduced-density operators $d_{1}$ and $d_{2}$ for the first and second spin from Eq. (44). From this equation we have

$$
\begin{aligned}
& d_{1}=\exp \left(-L_{1}-P_{1} Q_{1}^{\prime}\right), \\
& d_{2}=\exp \left(-L_{2}-P_{2} Q_{2}^{\prime}\right),
\end{aligned}
$$

where

$$
\begin{aligned}
& Q_{1}^{\prime}=\operatorname{Tr}_{2}\left(H K_{12} d_{2}\right), \\
& Q_{2}^{\prime}=\operatorname{Tr}_{1}\left(H K_{12} d_{1}\right) .
\end{aligned}
$$

According to Eq. (22), the correlation superoperator $K_{12}$, is defined through the relation:

$$
d=K_{12} d_{1} \otimes d_{2} \text {. }
$$

We will show in the following that the set of Eqs. (78) and (79) satisfy the exact reduced density operators $\tilde{d}_{1}$ and $\tilde{d}_{2}$ given by equations $(75)$ and (76). To do this we should use the expression

$$
\begin{aligned}
& d d_{1}^{-1}=K_{12} d_{2}, \\
& d d_{2}^{-1}=K_{12} d_{1}, \\
& d_{1} d_{1}^{-1}=l_{1}, \\
& d_{2} d_{2}^{-1}=l_{2} .
\end{aligned}
$$

After some simple calculations we have

$$
\begin{aligned}
& Q_{1}^{\prime}=\operatorname{Tr}_{2}\left(H d d_{1}^{-1}\right)=E_{3} d_{1} d_{1}^{-1}=E_{3} l_{1}, \\
& Q_{2}^{\prime}=\operatorname{Tr}_{1}\left(H d d_{2}^{-1}\right)=E_{3} d_{2} d_{2}^{-1}=E_{3} l_{2}, \\
& d_{1}=\exp \left(-l_{1}-P_{1} E_{3}\right), \\
& d_{2}=\exp \left(-l_{2}-P_{2} E_{3}\right) .
\end{aligned}
$$

From normalization conditions we have

$$
\begin{aligned}
& L_{1}=\ln 2-P_{1} E_{3}, \\
& L_{2}=\ln 2-P_{2} E_{3} .
\end{aligned}
$$

On the basis of Eqs. (87)-(92) and the condition of completeness we can easily prove that the following expressions are true:

$$
d_{1}=\frac{1}{2} l_{1}=\frac{1}{2}\left(|-1\rangle_{11}\langle-1|+| 1\rangle_{11}\langle 1|\right)=\tilde{d}_{1}
$$

and

$$
d_{2}=\frac{1}{2} l_{2}=\frac{1}{2}\left(|-1\rangle_{2}{ }_{2}\langle-1|+| 1\rangle_{2}{ }_{2}\langle 1|\right)=\tilde{d}_{2} .
$$

In this way we have proved that the reduced-density operators $d_{1}$ and $d_{2}$ obtained from Eq. (44) are identical with the corresponding operators obtained from exact calculations. Thus, we have shown that the proposed formalism is absolutely correct in two extreme cases of macroscopic systems and microscopic systems allowing exact solutions.

The results obtained for the two extreme cases prove the correctness of the assumptions made but are of little interest from the physical point of view, so in the following we shall deal with a construction of a quan- 
tum statistical operator and reduced-density operator for nanoscopic systems interacting with macroscopic environment. Now we describe two different methods taking into account the interaction between a nanoscopic system (or an ultrathin film) and the macroscopic substrate. In the first the problem is tackled in the terms of the QST, while the second is based on reduced-density operator (44).

\subsection{QST of a nanoscopic system}

The starting point of QST is that any macroscopic system because of very high mean density of states cannot be treated as isolated from its environment. The QST assumes that the interaction of a given macroscopic system with the environment is so weak that it can be described by vector of state $|\Psi(t)\rangle$ depending on time $t$. As a result of the environment effect the system is not one of the eigenstates $|E\rangle$ of the Hamiltonian $H$ :

$$
H|E\rangle=E|E\rangle,
$$

but in a non-stationary state depending on time. Under such an assumption the expectation value of any observable $a$ is determined by

$$
\langle a(t)\rangle=\langle\Psi(t)|a| \Psi(t)\rangle .
$$

A result of measurement during $\tau$ is the quantum state $|\Psi(t)\rangle$ averaged over a particular time interval $\langle 0, \tau\rangle$. Hence the expectation value reads

$$
\langle\langle a\rangle\rangle=\sum_{E} \sum_{E^{\prime}} C_{E, E^{\prime}}\left\langle E|a| E^{\prime}\right\rangle,
$$

where

$$
C_{E, E^{\prime}}=\frac{1}{\tau} \int_{0}^{\tau} \mathrm{d} t\langle\Psi(t) \mid E\rangle\left\langle E^{\prime} \mid \Psi(t)\right\rangle .
$$

Another assumption is that the disturbances of the macroscopic system caused by the environment are absolutely random, which leads to cancellation of the nondiagonal terms in Eq. (97), and

$$
C_{E, E^{\prime}}=p_{E} \delta_{E, E^{\prime}}
$$

where

$$
p_{E}=\frac{1}{\tau} \int_{0}^{\tau} \mathrm{d} t|\langle E \mid \Psi(t)\rangle|^{2} .
$$

Hence,

$$
\langle\langle a\rangle\rangle=\sum_{E} p_{E}\langle E|a| E\rangle=\operatorname{Tr}(a \rho),
$$

where $\rho$ is the statistical operator,

$$
\rho=\sum_{E}|E\rangle p_{E}\langle E|,
$$

and $p_{E}$ is the probability distribution determined assuming different statistical hypotheses that are beyond the quantum theory. Therefore there exist different approaches to the QST [6, 7]. It is known that a wide class of physical phenomena in the macroscopic systems being in the state of thermodynamical equilibrium can be sufficiently well described by the statistical operator [6]:

$$
\rho=\exp (\beta(F-H))=\sum_{E}|E\rangle \mathrm{e}^{\beta(F-E)}\langle E|,
$$

where

$$
F=-\frac{1}{\beta} \ln \operatorname{Tr}\left(\mathrm{e}^{-\beta H}\right),
$$

and $\beta=\left(k_{\mathrm{B}} T\right)^{-1}$.

In order to describe a nanoscopic system in terms of QST a macroscopic system is divided into a nanoscopic subsystem $j$ surrounded by the macroscopic part of the system $j^{\prime}$. After such a division the Hamiltonian $H$ can be expressed as

$$
H=H_{j}+H_{j^{\prime}}+H_{j j^{\prime}},
$$
where $H_{j}$ and $H_{j^{\prime}}$ are the Hamiltonians of the systems $j$ and $j^{\prime}$, while $H_{j j^{\prime}}$ is the term describing the interactions between these systems. Let $a_{j}$ be an operator corresponding to the observable describing the nanoscopic system $j$. The expectation value of this observable is given by

$$
\left\langle\left\langle a_{j}\right\rangle\right\rangle_{j}=\operatorname{Tr}\left(a_{j} \mathrm{e}^{\beta(F-H)}\right)=\operatorname{Tr}_{j}\left(a_{j} \rho_{j}\right)
$$

where $\rho_{j}$ is the reduced statistical operator of the nanoscopic system $j[1,4]$ :

$$
\rho_{j}=\operatorname{Tr}_{j^{\prime}}\left(\exp \left(\beta\left(F-H_{j}-H_{j^{\prime}}-H_{j j^{\prime}}\right)\right)\right),
$$

$\operatorname{Tr}_{j}(\ldots)$ and $\operatorname{Tr}_{j^{\prime}}(\ldots)$ a partial traces over the states of the systems $j$ and $j^{\prime}$, respectively. Expanding Eq. (114) into a power series used in the thermodynamic perturbation calculus [8], one obtains

$$
\rho_{j}=\mathrm{e}^{\beta\left(F_{j}-H_{j}\right)}\left\langle\left\langle U_{j j^{\prime}}\right\rangle\right\rangle_{j^{\prime}},
$$

where

$$
\begin{aligned}
& \langle\langle\ldots\rangle\rangle_{j^{\prime}}=\operatorname{Tr}_{j^{\prime}}\left(\ldots \mathrm{e}^{\beta\left(F_{j^{\prime}}-H_{j^{\prime}}\right)}\right), \\
& U_{j j^{\prime}}=P \exp \left(-\int_{0}^{\beta} \mathrm{d} \beta^{\prime} \tilde{H}_{j j^{\prime}}\left(\beta^{\prime}\right)\right), \\
& \tilde{H}_{j j^{\prime}}=\mathrm{e}^{\beta\left(H_{j}+H_{j^{\prime}}\right)} H_{j j^{\prime}} \mathrm{e}^{-\beta\left(H_{j}+H_{j^{\prime}}\right)}, \\
& F_{j^{\prime}}=-\frac{1}{\beta} \ln \operatorname{Tr}_{j^{\prime}}\left(\mathrm{e}^{\left.-\beta H_{j^{\prime}}\right),}\right. \\
& F_{j}=-\frac{1}{\beta} \ln \operatorname{Tr}_{j}\left(\mathrm{e}^{\left.-\beta H_{j}\left\langle\left\langle U_{j j^{\prime}}\right\rangle\right\rangle_{j^{\prime}}\right)}\right.
\end{aligned}
$$

and $P$ is the operator analogous to the Dyson timeordering operator. Equations (106) and (108) permit a full description of the physical properties of a nanoscopic system $j$ in the state of the thermodynamic equilibrium and interacting with its environment.

According to Eqs. (106) and (108) we obtained expression for expectation value of an arbitrary observable $a$ of the nanoscopic system $j$ :

$$
\left\langle\left\langle a_{j}\right\rangle\right\rangle_{j}=\operatorname{Tr}_{j}\left(a_{j} \mathrm{e}^{\beta\left(F_{j}-H_{j}\right)}\left\langle\left\langle U_{j j^{\prime}}\right\rangle\right\rangle_{j^{\prime}}\right) .
$$




\subsection{Reduced-density operator for nanoscopic system}

In contemporary science and technology we very often deal with multiparticle systems which cannot be considered as massive macroscopic systems. For example they are ultrathin multilayer systems, mesoscopic or nanoscopic systems or many of biological systems. They are most often met e.g. in micro- and nanoelectronic devices. Because of their unique physical characteristics, they are referred to by the new terms mezophysics and nanophysics which appeared in literature.

From the point of view of our considerations, the important feature of these systems is that their interaction with environment is not a small surface effect, and therefore, the terms describing their interaction with environment cannot be neglected. Let us study a nanoscopic system $j$ surrounded by a massive macroscopic system $j^{\prime}$. Thus, the system $j+j^{\prime}$ is a massive macroscopic system whose interaction with the remaining part of the universe can be neglected.

In analogy to the formula for the reduced-density operator (48) we can write the reduced density operator for the system $j+j^{\prime}$ in the form

$$
d_{j+j^{\prime}}=\exp \left(-L_{j+j^{\prime}}-\underline{P}_{j+j^{\prime}} \underline{Q}_{j+j^{\prime}}\right)
$$

and for the system $j^{\prime}$ :

$$
d_{j^{\prime}}=\exp \left(-L_{j^{\prime}}-\underline{P}_{j^{\prime}} \underline{Q}_{j^{\prime}}\right) .
$$

For the system $j$, the operator (44) takes the form

$$
d_{j}=\exp \left(-L_{j}-\underline{P}_{j}\left(\underline{Q}_{j}+\underline{Q}_{j}^{\prime}\right)\right),
$$

where

$$
\begin{aligned}
& \underline{Q}_{j}^{\prime}=\operatorname{Tr}_{j^{\prime}}\left(\underline{Q}_{j j^{\prime}} \operatorname{Tr}_{R^{\prime}}\left(K_{j^{\prime \prime} \neq j, j^{\prime}}^{\otimes} d_{j^{\prime \prime}}\right) d_{j^{\prime}}\right)= \\
& \quad \operatorname{Tr}_{j^{\prime}}\left(\underline{Q}_{j j^{\prime}} K_{j j^{\prime}} d_{j^{\prime}}\right)
\end{aligned}
$$

and $K_{j j^{\prime}}$ is the reduced-correlation superoperator between systems $j$ and $j^{\prime}$, defined by the following formula (see Eq. (22)):

$$
\begin{aligned}
& d_{j+j^{\prime}}:=K_{j j^{\prime}} d_{j} \otimes d_{j^{\prime}} \equiv\left(1_{j+j^{\prime}}+\kappa_{j j^{\prime}}\right) d_{j} \otimes d_{j^{\prime}} \equiv \\
& \operatorname{Tr}_{R^{\prime}}\left(K_{j^{\prime \prime} \neq j, j^{\prime}} d_{j^{\prime \prime}}\right) d_{j} \otimes d_{j^{\prime}}, \\
& K_{j j^{\prime}}=\operatorname{Tr}_{R^{\prime}}\left(K_{\substack{j^{\prime \prime} \neq j, j^{\prime} \\
j_{j^{\prime \prime}}}}^{\otimes} d_{j^{\prime \prime}} .\right.
\end{aligned}
$$

Now we shall find the explicit although formal equation for the reduced-correlation operator, $\kappa_{j j^{\prime}}$ and then the reduced-correlation superoperator $K_{j j^{\prime}}$. Since the operator (115) is a function of the operator $\underline{Q}_{j+j^{\prime}}$, these operators commute, so

$$
\bar{Q}_{j+j^{\prime}} d_{j+j^{\prime}}=\underline{0},
$$

where $\underline{\bar{Q}}_{j+j^{\prime}}$ is the commutator superoperator

$$
\underline{Q}_{j+j^{\prime}}:=\left[\underline{Q}_{j+j^{\prime}},\right]_{-}
$$

and the brackets [, ] stand for the commutator. For the same reasons the following relation holds:

$$
\bar{Q}_{j^{\prime}} d_{j^{\prime}}=\underline{0}
$$

where

$$
\bar{Q}_{j^{\prime}}:=\left[\underline{Q}_{j^{\prime}},\right]_{-} .
$$

As follows from the formula (15) we have

$$
\underline{Q}_{j+j^{\prime}}=\underline{\bar{Q}}_{j}+\underline{Q}_{j^{\prime}}+\underline{\bar{Q}}_{j j^{\prime}},
$$

where

$$
\begin{aligned}
& \underline{\bar{Q}}_{j}:=\left[\underline{Q}_{j},\right]_{-}, \\
& \bar{Q}_{j j^{\prime}}:=\left[\underline{Q}_{j j^{\prime}},\right]_{-} .
\end{aligned}
$$

Putting the expressions (119) and (124) into (102) and using Eq. (122), we get

$$
\left(\underline{\bar{Q}}_{j}+\underline{\bar{Q}}_{j j^{\prime}}\right) d_{j} \otimes d_{j^{\prime}}+\underline{\bar{Q}}_{j+j^{\prime}} \kappa_{j j^{\prime}} d_{j} \otimes d_{j^{\prime}}=\underline{0} .
$$

Introducing the superoperator $\underline{Q}_{j+j^{\prime}}^{-1}$ inverse to the superoperator $\underline{\bar{Q}}_{j+j^{\prime}}$, defined as:

$$
\underline{\bar{Q}}_{j+j^{\prime}}^{-1} \underline{\bar{Q}}_{j+j^{\prime}}=1_{j+j^{\prime}}
$$

we can write

$$
\kappa_{j j^{\prime}}=-\bar{Q}_{j+j^{\prime}}^{-1}\left(\underline{Q}_{j}+\underline{Q}_{j j^{\prime}}\right)
$$

and, as follows from Eq. (119):

$$
K_{j j^{\prime}}=1_{j+j^{\prime}}-\underline{\bar{Q}}_{j+j^{\prime}}^{-1}\left(\underline{\bar{Q}}_{j}+\underline{Q}_{j j^{\prime}}\right) .
$$

The superoperators have their matrix representations [9] and thus it is possible to define a superoperator inverse to a given superoperator.

In this way we have obtained an explicit although formal expression for the reduced-correlations superoperator $K_{j j^{\prime}}$ between the systems $j$ and $j^{\prime}$ satisfying the condition (23).

Substituting Eq. (118), (130) and (116) into expression (117) we get

$d_{j}=$

$\exp \left(-L_{j}-\underline{P}_{j} \underline{Q}_{j}-\underline{P}_{j} \operatorname{Tr}_{j^{\prime}}\left(\underline{Q}_{j j^{\prime}} \exp \left(-L_{j^{\prime}}-\underline{P}_{j^{\prime}} \underline{Q}_{j^{\prime}}\right)\right)\right.$

$\left.+\underline{P}_{j} \operatorname{Tr}_{j^{\prime}}\left(\underline{Q}_{j j^{\prime}} \underline{\bar{Q}}_{j+j^{\prime}}^{-1}\left(\underline{\bar{Q}}_{j}+\underline{Q}_{j j^{\prime}}\right) \exp \left(-L_{j^{\prime}}-\underline{P}_{j^{\prime}} \underline{Q}_{j^{\prime}}\right)\right)\right)$.

The formula (131) is the most general form of the equilibrium reduced-density operator for any system $j$ interacting with its macroscopic environment $j^{\prime}$. It is more general than the formulae used in QST in the theory of macroscopic systems.

In the particular case when $j^{\prime}$ is described by the reduced-density operator (59), we get

$$
\begin{aligned}
& d_{j}=\exp \left(-L_{j}-\underline{P}_{j} \underline{Q}_{j}+P_{1 j} \operatorname{Tr}_{j^{\prime}}\left(H_{j j^{\prime}} \mathrm{e}^{\beta_{j^{\prime}}\left(F_{j^{\prime}}-H_{j^{\prime}}\right)}\right)\right. \\
& \left.+P_{1 j} \operatorname{Tr}_{j^{\prime}}\left(H_{j j^{\prime}} \underline{Q}_{j+j^{\prime}}^{-1}\left(\underline{Q}_{j}+\underline{Q}_{j j^{\prime}}\right) \mathrm{e}^{\beta_{j^{\prime}}\left(F_{j^{\prime}}-H_{j^{\prime}}\right)}\right)\right) .
\end{aligned}
$$

When the system $j$ contains a sufficiently large number of particles, we can neglect the reduced-correlation superoperator $\kappa_{j j^{\prime}}$ in Eq. (131) and then, instead of (132) we get 
$d_{j}=\exp \left(-L_{j}-\underline{P}_{j} \underline{Q}_{j}-P_{1 j} \operatorname{Tr}_{j}\left(H_{j j^{\prime}} \mathrm{e}^{\beta_{j^{\prime}}\left(F_{j^{\prime}}-H_{j^{\prime}}\right)}\right)\right)$

Moreover, assuming that the system $j$ can exchange only energy with the system $j^{\prime}$, the reduced-density operator takes the form

$$
\begin{aligned}
d_{j} & =\exp \left(\beta _ { j } \left(F_{j}-H_{j}-\operatorname{Tr}_{j^{\prime}}\left(H_{j j^{\prime}} \mathrm{e}^{\beta_{j^{\prime}}\left(F_{j^{\prime}}-H_{j^{\prime}}\right)}\right)\right.\right. \\
& \left.\left.+\operatorname{Tr}_{j^{\prime}}\left(H_{j j^{\prime}} \underline{\bar{Q}}_{j+j^{\prime}}^{-1}\left(\underline{\bar{Q}}_{j}+\underline{\bar{Q}}_{j j^{\prime}}\right) \mathrm{e}^{\beta_{j^{\prime}}\left(F_{j^{\prime}}-H_{j^{\prime}}\right)}\right)\right)\right),
\end{aligned}
$$

where from the normalization conditions we get

$$
\begin{aligned}
& F_{j^{\prime}}=-\frac{1}{\beta_{j^{\prime}}} \ln Z_{j^{\prime}}, \\
& F_{j}=-\frac{1}{\beta_{j}} \ln Z_{j}, \\
& Z_{j^{\prime}}=\operatorname{Tr}_{j^{\prime}}\left(\mathrm{e}^{-\beta_{j^{\prime}} H_{j^{\prime}}}\right), \\
& Z_{j}=\operatorname{Tr}_{j}\left(\operatorname { e x p } \left(\beta _ { j } \left(-H_{j}-\operatorname{Tr}_{j^{\prime}}\left(H_{j j^{\prime}} \mathrm{e}^{\beta_{j^{\prime}}\left(F_{j^{\prime}}-H_{j^{\prime}}\right)}\right)\right.\right.\right. \\
& \left.\left.\left.+\operatorname{Tr}_{j^{\prime}}\left(H_{j j^{\prime}} \bar{Q}_{j+j^{\prime}}^{-1}\left(\underline{Q}_{j}+\underline{Q}_{j j^{\prime}}\right) \mathrm{e}^{\beta_{j^{\prime}}\left(F_{j^{\prime}}-H_{j^{\prime}}\right)}\right)\right)\right)\right) .
\end{aligned}
$$

The reduced-density operator (134) for $\beta_{j^{\prime}}=\beta_{j}$ can be expressed as

$$
d_{1}=\exp \left(\beta_{j}\left(F_{j}-H_{j}-H_{j}^{\prime}\right)\right),
$$

where

$$
H_{j}^{\prime}=\operatorname{Tr}_{j^{\prime}}\left(H_{j j^{\prime}} K_{j j^{\prime}} d_{j^{\prime}}\right)
$$

and

$$
d_{j^{\prime}}=\exp \left(\beta_{j}\left(F_{j^{\prime}}-H_{j j^{\prime}}\right)\right) .
$$

From expression (119) we get

$$
\begin{aligned}
& d_{j+j^{\prime}}=K_{12} d_{j} \otimes d_{j^{\prime}} \cong K_{j j^{\prime}} d_{j^{\prime}} \otimes d_{j}^{o}, \\
& K_{12}=d_{j+j^{\prime}}\left(d_{j}^{o}\right)^{-1},
\end{aligned}
$$

where

$$
d_{j}^{o}=\exp \left(\beta_{j}\left(F_{j}-H_{j}\right)\right)
$$

and

$$
\left(d_{j}^{o}\right)^{-1}=\exp \left(-\beta_{j}\left(F_{j}-H_{j}\right)\right)
$$

is operator inverse to the operator $d_{j}^{o}$.

Substituting Eqs. (143) and (145) to (140) and after calculations we arrive at

$$
H_{j}^{\prime}=\frac{\left\langle H_{j j^{\prime}} V_{j j^{\prime}}\right\rangle_{j^{\prime}}}{\left\langle V_{j j^{\prime}}\right\rangle_{j+j^{\prime}}^{o}}
$$

where

$$
\begin{aligned}
& \langle\ldots\rangle_{j^{\prime}}=\operatorname{Tr}_{j^{\prime}}\left(\ldots d_{j^{\prime}}\right), \\
& \langle\ldots\rangle_{j+j^{\prime}}^{o}=\operatorname{Tr}_{j+j^{\prime}}\left(\ldots d_{j+j^{\prime}}^{o}\right), \\
& d_{j+j^{\prime}}^{o}=\exp \left(\beta_{j}\left(F_{j+j^{\prime}}^{o}-H_{j+j^{\prime}}^{o}\right)\right),
\end{aligned}
$$

$$
\begin{aligned}
& H_{j+j^{\prime}}^{o}=H_{j}+H_{j^{\prime}}, \\
& V_{j j^{\prime}}=1-\int_{0}^{\beta_{j}} \mathrm{~d} \beta^{\prime} V_{j j^{\prime}} \tilde{H}_{j j^{\prime}}, \\
& \tilde{H}_{j j^{\prime}}=\exp \left(-\beta^{\prime} H_{j+j^{\prime}}^{o}\right) H_{j j^{\prime}} \exp \left(\beta^{\prime} H_{j+j^{\prime}}^{o}\right) .
\end{aligned}
$$

Using Eq. (151) we can approximate operator $V_{j j^{\prime}}$ by the method of iteration with the first approximation expressed as

$$
V_{j j^{\prime}}=1-\int_{0}^{\beta_{j}} \mathrm{~d} \beta^{\prime} \tilde{H}_{j j^{\prime}}
$$

Expanding $V_{j j^{\prime}}$ into a power series analogously used in the thermodynamical perturbation calculus [8], one obtains

$$
V_{j j^{\prime}}=P \exp \left(-\int_{0}^{\beta_{j}} \mathrm{~d} \beta^{\prime} \tilde{H}_{j j^{\prime}}\right),
$$

where $P$ is the operator analogous to the Dyson timeordering operator.

According to Eqs. (18), (108), (139) and (146) we obtained two different expressions for expectation value of an arbitrary observable $a_{j}$ of the nanoscopic system $j$ :

$$
\left\langle a_{j}\right\rangle=\operatorname{Tr}_{j}\left(a_{j} \exp \left(\beta_{j}\left(F_{j}-H_{j}\left(\beta_{j}\right)\right)\right)\right)
$$

and (114), where

$$
H_{j}\left(\beta_{j}\right)=H_{j}+\frac{\left\langle H_{j j^{\prime}} V_{j j^{\prime}}\right\rangle_{j^{\prime}}}{\left\langle V_{j j^{\prime}}\right\rangle_{j+j^{\prime}}^{o}} .
$$

Equation (155) is easier in specific applications than (114) because it takes into account the interactions with environment through an easy replacement of the Hamiltonian $H_{j}$ of a nanoscopic system $j$ by the effective Hamiltonian $H_{j}\left(\beta_{j}\right): H_{j} \rightarrow H_{j}\left(\beta_{j}\right)$.

\section{Conclusions}

Equation (47) is the most general form of the fundamental equation of the thermodynamics of equilibrium states. When we neglect the terms describing the interaction of a given system with its environment this equation takes the form of the fundamental equation of the QST. As we have shown, Eq. (47) applied to a model exactly solvable microscopic system also leads to exact results.

This equation has been derived on the basis of the quantum theory variational principle and not the QST, so without statistical hypotheses. Therefore, the approach leading to Eq. (47) could be called quantum thermodynamics (QT) to distinguish it from the QST.

The QT is conceptually simpler and more general than the QST. It is not only suitable for description of macroscopic systems (like QST) but it can be also applied to describe nanoscpic, mezoscopic systems, and ultrathin films. Moreover, in the QT it can be proved that the dependence of the equilibrium reduced-density operators 
(or statistical operators) on non-additive operators commuting with the Hamiltonian can be disregarded, which is assumed in the quantum statistical mechanics.

We have shown that fundamentals of the quantum thermodynamics do not have to be formulated in analogy to the classical statistical thermodynamics, because we do not have to supplement the quantum theory with the statistical methods as the probabilistic concepts are inherent in the quantum theory. The quantum theory is holistic theory, which reflects the fact that, according to the fundamental postulate to the quantum cosmology, the universe has a vector of state, common to all its macroscopic many particle subsystems, and vectors of state of its particular subsystems cannot be specified. In the classical description we can simultaneously have full information about the state of a whole system and its particular components. The holistic properties of the quantum theory allow a comprehensive description of many particle systems in the formalism of reduceddensity operators.

The only postulate used in the quantum thermodynamics that goes beyond the frames of quantum cosmology is the assumption that the universe can be divided into parts whose state practically does not change in time - the postulate of equilibrium. This postulate has the character of an assumption specifying the states of the universe subsystems and is in agreement with the facts observed in reality. As it follows from our experience, most often many-particle systems being in the environment undergoing slow time changes, reach the equilibrium state sooner or later. After they have reached this state we can divide the universe into at least two parts $k=2$ being in the equilibrium state: a given manyparticle subsystem and the remaining part of the universe. The postulate claiming the possibility of the division of the universe into $k \geq 2$ parts being in the state of equilibrium does not limit the general character of the principles of the quantum thermodynamics, which simply is the quantum theory of many-particle systems in the equilibrium states. Thus the problem of falsification of the quantum thermodynamics is therefore at the same level of generalisation as that of falsification of the quantum cosmology.
The reduced-density operator (133) takes into account the interaction with environment only in the mean field type approximation and that is why it is much easier in applications than the reduced statistical operator (131) describing this interaction in a complete form. The simplified form of the reduced-density operator (133) has been successfully used for description of the influence of substrate on the properties of the magnetic ultrathin films [10] and spin nanoscopic systems $[11,12]$. The authors of these works have proved that in many cases the interaction with substrate significantly affects the physical properties of such systems.

Therefore, in future, such systems should be described using the non-simplified form of the reduced-density operator (131). This operator may also be useful in description of certain devices appearing in quickly developing quantum engineering and quantum technology, molecular electronics, and biological systems studied in molecular biophysics.

\section{References}

[1] Z. Onyszkiewicz, Phys. Status Solidi B 150, 109 (1988).

[2] Z. Onyszkiewicz, Phys. Lett. A 163, 450 (1992).

[3] Z. Jacyna-Onyszkiewicz, Physica A 305, 497 (2002).

[4] Z. Jacyna-Onyszkiewicz, Quantum Cosmogenesis, The Uni-Publications, Bayreuth 2012.

[5] K. Blum, Density Matrix, Theory and Applications, Plenum, New York 1981.

[6] Z. Jacyna-Onyszkiewicz, The Principles of Quantum Thermodynamics, A. Mickiewicz University Publisher, Poznań 1996 (in Polish).

[7] R. Balescu, Equilibrium and Nonequilibrium Statistical Mechanics, Wiley, New York 1975.

[8] T. Matsubara, Prog. Theor. Phys. 14, 351 (1955).

[9] N.H. Ernst, G. Bodenhausen, A. Woukaun, Principles of Nuclear Magnetic Resonance in One and Two Dimensions, Clarendon Press, Oxford 1987.

[10] W. Jaworski, Acta Phys. Pol. A 115, 171 (2009).

[11] L.S. Borkowski, Z. Jacyna-Onyszkiewicz, Acta Phys. Pol. A 112, 1223 (2007); Acta Phys. Pol. A 115, 375 (2009).

[12] L.S. Borkowski, Z. Jacyna-Onyszkiewicz, Surf. Sci. 603, 27 (2009). 